\title{
Design on jQuery Evaluation System Framework of Movements Resource
}

\author{
Yuanhai Wang \\ School Physical Education, Yuxi Normal University, Yuxi, 653100, China
}

Keywords: jQuery framework, Management evaluation system, Simulation analysis

\begin{abstract}
. movements association is voluntary participation, public non-profit social organizations. With the continuous development of social economy and improvement of people's living level, more and more people choose to join the movements club. At present, many movements associations have shortcomings in the activities organization and financial management. This paper firstly introduces the concept of jQuery framework, and then constructs the movements community management evaluation system based on jQuery framework, finally doses simulation analysis on the overall performance and the evaluation performance. The results show the movements community management and evaluation system can guide community staffing, activity organization and fiscal expenditure, which has good guidance and reference for the better development of movements associations.
\end{abstract}

\section{Introduction}

jQuery is created by John Resig in 2006 , which is prototype is a more optimized Javascript framework after prototype. jQuery framework simplifies the preparation process of JavaScript and Ajax in a certain extent [2]. It also allows users to operate the document in the webpage, handle events, implementation effects and add the Ajax interactive program for all webpages. The jQuery framework has the following characteristics: strong function storage; solving browser compatibility issues for different versions; more rich animation effects; automatically correcting the wrong script information; rich plug-in.The operation of dom is simple, in the operation it is involved in the selection of jQuery set. The function mainly includes selectors and filters, as shown below:

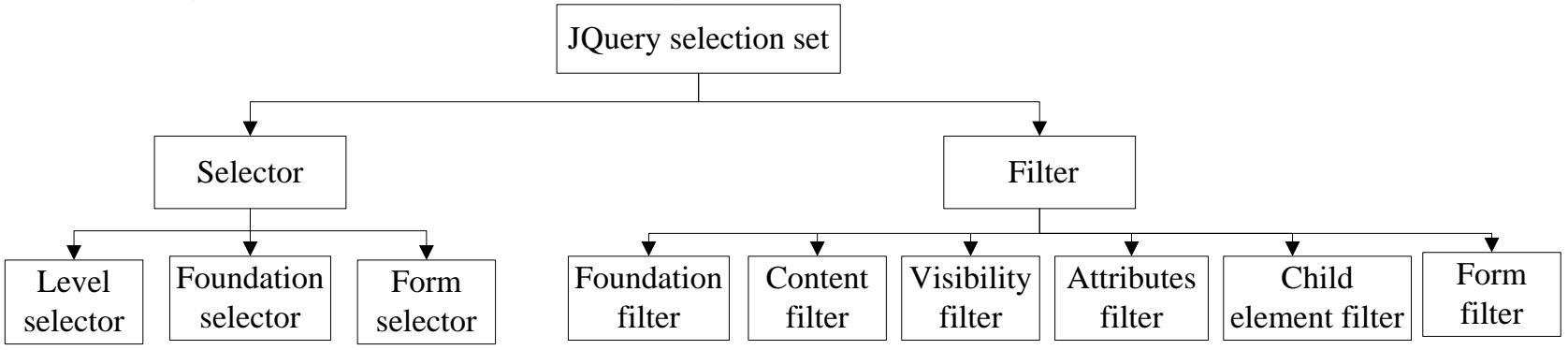

Fig. 1 Schematic diagram of jQuery selection set

As shown in Figure 1, the jQuery's selection set is powerful, including selectors and filters. The selector function includes level selector, foundation selector and form selector [3]. The function of filter includes foundation filter, content filter, visibility filter, attributes filter, sub-elements filter, form filter.

\section{Design of movements Community Management Evaluation System based on jQuery Framework}

Because the development of movements organizations in China is still at the starting stage, policies, regulations and management measures are not very in place, there are still some problems such as non-uniform activities, non fixed staff and the fiscal mess in the movements community management [4]. The movements community management and evaluation system includes front user end and background management end. The front user end includes user authentication, user messages, 
activities information display [5, 6]. The backstage management includes user management, message processing, financial management.

The first reference 'script src= "jquery.min.js" >' /script ', then put it into the jQuery framework, thereby obtaining the jQuery selector and a variety of functions, the first step of the jQuery program is:

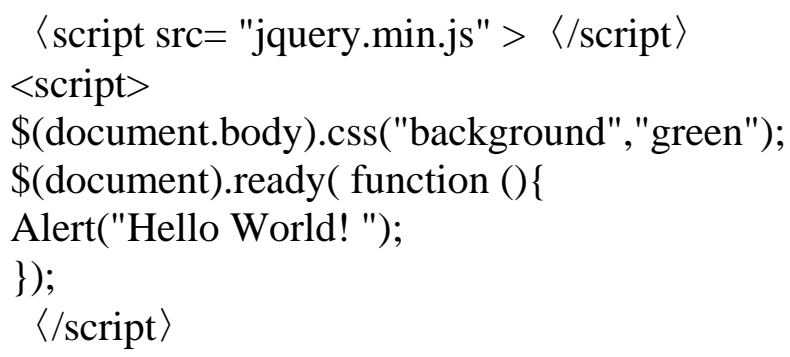

Then doing bean configuration on the user data source, as follows:

$<$ bean id="dataSource"

class ="org.apache.commons.dbcp.BasicDataSource"

destroy-method="close">

〈property nanie $=$ "driverClassName" value $=$ "

com.microsoft.sqlserver.jdbc.SQLServerDriver " />

〈property name="urr* value: "

jdbc:sqlserver://localhost:1238;database=bookshop" />

〈property name="usemame" value: "sa" />

$\langle$ property name="password" value="777888" />

$<$ /bean>

The above code is the running program process of the movements community management evaluation system based on the jQuery framework. The system operation program is simple, and the computer configuration requirement is not high, but also convenient for user operation [8,9]. It has very great help for personnel management, financial management of movements community affairs management.

\section{Simulation Analysis}

The computer simulation system has wide application in the system stability analysis based on the impulse response function, and the result is really reliable. The simulation analysis process is as follows:

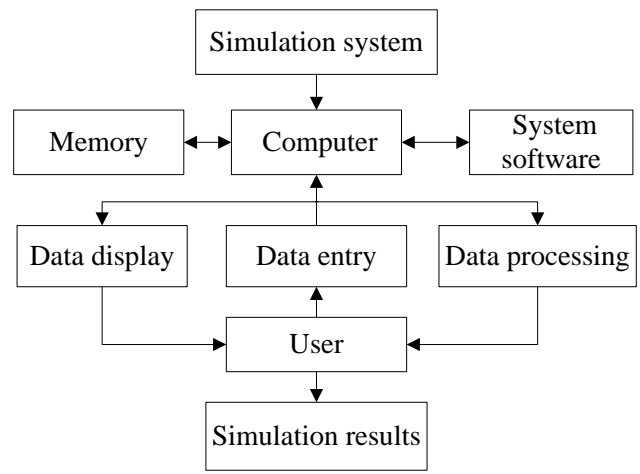

Fig. 2 Schematic diagram of simulation system operation

As shown in Figure 2, the computer simulation system mainly includes computer, storage, and system software [10]. The users put the simulation data into the computer, and the computer will automatically process data. The simulation process is as follows:

Assuming that the impulse response function is convergent, so $\lim _{t \rightarrow \infty} k(t)=0$. It shows that the evaluation system is stable. 
When $\lim _{t \rightarrow \infty} k(t)=1$, the pulse response of system is a closed loop transfer, so the closed-loop transfer function can be set:

$\Phi(s)=\frac{M(s)}{D(s)}=\frac{b_{m}\left(s-z_{1}\right)\left(s-z_{2}\right) \cdots\left(s-z_{m}\right)}{a_{n}\left(s-\lambda_{1}\right)\left(s-\lambda_{2}\right) \cdots\left(s-\lambda_{n}\right)}$.

$z_{1}, z_{2}, \cdots, z_{m}$ are starting points of the closed loop, $\lambda_{1}, \lambda_{2}, \cdots, \lambda_{n}$ are end points of the closed loop.

The impulse response function is:

$C(s)=\Phi(s)=\frac{b_{m}\left(s-z_{1}\right) \cdots\left(s-z_{m}\right)}{a_{n}\left(s-\lambda_{1}\right) \cdots\left(s-\lambda_{n}\right)}$

Changing the formula (2) into:

$C(s)=\frac{A_{1}}{s-\lambda_{1}}+\frac{A_{2}}{s-\lambda_{2}}+\cdots+\frac{A_{n}}{s-\lambda_{n}}=\sum_{i=1}^{n} \frac{A_{i}}{s-\lambda_{i}}$.

The unit impulse function can be obtained through rush transform for formula (3).

$k(t)=\sum_{i=1}^{n} A_{i} e^{\lambda_{i} t}$

When $\lambda_{i}$ is the conjugate complex roots, $\lambda_{i}=\sigma_{i} \pm j \omega_{i}$. Then formula (4) can be expressed as: $A_{i} e^{\left(\sigma_{i}+j \omega_{i}\right) t}+A_{i+1} e^{\left(\sigma_{i}-j \omega_{i}\right) t}$ or $A e^{\sigma_{i} t} \sin \left(\omega_{i} t+\psi_{i}\right)$.

So it can decide if the system is stable through three conditions: $\lambda_{i}<0$ shows system is stable; $\lambda_{i}>0$ shows system is not stable; $\lambda_{i}=0$ shows the system is at the critical point, not stable.

The above formula (1), (2), (3) and (4) are the operational process of impulse response function. The operation process is more complex in practice. In order to further simplify the operation, it can use Routh algebra criterion. The function expression is:

$D(s)=a_{n} s^{n}+a_{n-1} s^{n-1}+\cdots+a_{1} s+a_{0}=0$.

When $a_{i}>0$, it shows simulation system is stable, otherwise is not stable.

Through the above function operation process, the simulation results can be obtained as shown in Figure 3.

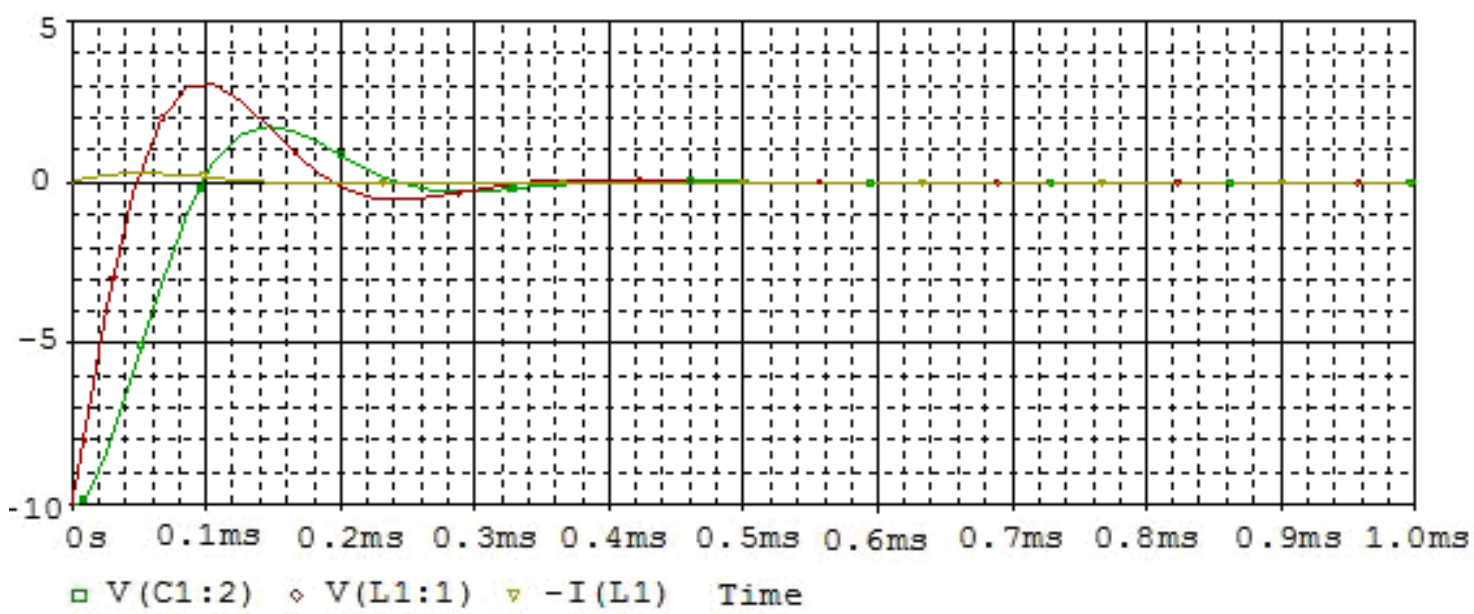

Fig. 3 Schematic diagram of the simulation results

As shown in Figure 3, in the initial stage of the simulation test $(0-0.3 \mathrm{~min}) \lim _{t \rightarrow \infty} k(t) \neq 0$, the system is not stable. After $0.3 \min , \lim _{t \rightarrow \infty} k(t)=0$, the system has been in steady state[11]. Therefore the stability of the movements community management evaluation system based on jQuery framework is good, which play an irreplaceable role in accelerating the development of movements organizations and improving the level of movements association community management. 


\section{Summary}

At present most of the movements community in China has the problem of not fixed staff, single activities form and financial management confusion. This paper constructs movements community management and evaluation system based on jQuery framework and the operation is simple and running stable operation, which can greatly improves the efficiency and effectiveness of movements community management.

\section{References}

[1] Cai Shengbin. Problem and countermeasure for the social organizations [D]. Xiangtan University, 2011:3-17.

[2] Li Li, Zhang Lin. Public service of movements: movements development on public finance needs [J]. movements science, 2012, 30(6): 53-80.

[3] Hou Xiaofu. Open another door - present situation and development of Chinese organizations [M]. The mass press, 2011: 56-80.

[4] Lu Yuanzhen. Journal of movements community [J]. Journal of Beijing Sport University, 2012, 19(1): 2-3.

[5] Li Zhixiu, Zhang Jun, Chen Guang, Yang Lihong. Application of jQueryAjax asynchronous processing Json data in project management system [J]. Journal of Yunnan University, 2011, 33(2): 247-255.

[6] Richard York (USA). The classic of jQuery JavaScript and CSS developing [M]. Beijing: Tsinghua University press, 2011: 102-123.

[7] Han Guanglin. Design and implementation of the university graduate management system based on B/S mode [D]. Nankai University, 2012: 2-15.

[8]Timothy J. Rowley. Moving Beyond Dyadic Ties: A Network Theory of Stakeholder Influences [J]. The Academy of Management Review, 2011, 22(4): 887-900.

[9] J.Wojeiechowski, B.Sakowiez, K.Dura, MVCModel. Struts Framework and File upload Issues in Web APPIieations Based on J2EE Platform [J]. TCSESOCM, 2011, 3(4): 1102-1112.

[10]Reinhard Klemm. Efficient Dynamic Multicity in Enterprise JavaBeans [C]. Proceedings of the 11th IEEE International Conference on Engineering of Complex Computer Systems, 2011: 670-679.

[11]DanLi, Chongquan Zhong, Xiaofeng Yuan, Li Zhang. Study on Embedded Equipment Web Monitoring and Control Based on Industrial Ethernet [C]. Proceedings of the 6th World Congress on Intelligent Control, 2011: 1320-1329. 